\title{
Mineração
}

\section{Flotação de diferentes tipologias de minério fosfático de Tapira/MG, usando o coletor óleo de soja hidrogenado}

\author{
Gilberto Caixeta Guimarães \\ Mestrando do PPGEM/UFOP e Professor do CEFET-OP. E-mail: caixeta@barroco.com.br \\ Rosa Malena Fernandes Lima \\ Dra., Professora do PPGEM/UFOP.E-mail: rosa@demim.ufop.br \\ Maria Lúcia Magalhães de Oliveira \\ Dra.,Professora do DEMIN/UFOP.E-mail:mlucia@demin.ufop.br
}

\section{Resumo}

Esse trabalho teve por objetivo avaliar o desempenho do coletor óleo de soja hidrogenado na flotação de quatro tipologias de minérios fosfáticos da mina de Tapi$\mathrm{ra} / \mathrm{MG}$, denominadas piroxenito silicificado semicompacto, piroxenito silicificado compacto, silexito e piroxenito serpentinizado, cujas relações $\mathrm{CaO} / \mathrm{P}_{2} \mathrm{O}_{5}$ eram de 2,1, 3,5, 2,5 e 1,33, respectivamente. Para a realização dos experimentos, foram aplicadas a metodologia estatística de planejamento e a análise fatorial de experimentos, onde foram estudadas as dosagens de coletor (óleo de soja), a dosagem de depressor (amido de milho gelatinizado) e o $\mathrm{pH}$ de flotação. Verificou-se que o valor de $\mathrm{pH}$ ideal para a flotação dos minérios foi igual a 11 , exceto para o minério silexito, que foi igual a 8 . A relação $\mathrm{CaO} / \mathrm{P}_{2} \mathrm{O}_{5}$ dos concentrados foi maior para os minérios com maior conteúdo de carbonatos e aumentava com o aumento da dosagem de coletor, evidenciando a baixa seletividade desse reagente para a separação entre a apatita e a ganga carbonática a ela associada. Observou-se a necessidade de etapa cleaner para obtenção de concentrados dentro das especificações de mercado para todas as amostras estudadas

Palavras-chave: rocha fosfática, flotação, seletividade entre apatita e calcita.

\begin{abstract}
In this work was studied the froth flotantion response of fuor diferent types of phosphate ores from Tapira-MG using fatty acid soap from soja as apatite colector and corn starch gelatinized as gangue depress. The $\mathrm{CaO} / \mathrm{P}_{2} \mathrm{O}_{5}$ relation of the half compact silificate piroxenite, compact silificate piroxenite, silexite and serpentinized piroxenite ores samples were 2,1, 3,5, 2,5 e 1,33, respectivally. The experiments were conduct and analised using fatorial planing of experiments that studied the influence of $\mathrm{pH}$, coletctor and starch concentration. The optimun $\mathrm{pH}$ value to float the sample ores was 11, except for silexite sample that it was 8. The increase of colector concentration conducts to concentrates with lower $\mathrm{P}_{2} \mathrm{O}_{5}$ content and higher $\mathrm{CaO} /$ $\mathrm{P}_{2} \mathrm{O}_{5}$ relation. The concentrates relation from ores with higher relation $\mathrm{CaO} / \mathrm{P}_{2} \mathrm{O}_{5}$ were higher too what confirme the poor selectivity among apatite and carbonates by fatty acid soap. In order to obtain the market chemical specifications of phophate concentrates is necessary the cleaner step for all ores samples studied.
\end{abstract}

Keywords: phosphate ore, flotation, selectivity. 


\section{Introdução}

Os principais minérios fosfatados brasileiros requerem um tratamento complexo e são de difícil benefíciamento por apresentarem mineralogia complexa, baixo grau de uniformidade, baixo teor de apatita e elevado teor de carbonatos, o que implica problemas no seu aproveitamento industrial. A lavra e o processamento desses minérios levam a perdas de fósforo, sendo $15 \%$ na lavra, $40 \%$ no beneficiamento, 2 a $5 \%$ no transporte e manuseio do concentrado fosfático. Finalmente, a assimilação do fósforo presente nos fertilizantes pelas plantas é muita baixa, ou seja, de 10 a 30\%. Esses índices levam a um aproveitamento global máximo de apenas $8 \%$ do fósforo inicial (Barros, 1997). Como pode ser observado, uma das maiores perdas ocorre na etapa de beneficiamento, exigindo, cada vez mais, aprimoramentos no processo de concentração para um melhor aproveitamento das reservas fosfáticas, contribuindo, assim, para o desenvolvimento sustentado.

Embora intensivos esforços na pesquisa tenham sido feitos para desenvolver métodos práticos para obter a separação carbonato/apatita através da flotação, um dos casos de sucesso industrial é do processo Serrana, aplicado para tratar um minério fosfático carbonático (15\% de apatita). O aspecto-chave do processo consistia no uso da flotação aniônica direta da apatita com ácidos graxos de "tall oil" saponificado e depressão do carbonato com amido de milho gelatinizado num circuito alcalino. $\mathrm{O}$ processo evoluiu para a substituição do coletor por um reagente anfótero da família da sarcozina. Outros estudos mostraram que a rugosidade da superfície, o tamanho dos grãos e a contaminação de ferro influenciam a flotabilidade de apatitas e carbonatos. O desenvolvimento desse processo ajudou a diminuir a dependência brasileira por fosfatos estrangeiros (Assis e colaboradores, 1988; Leal Filho e colaboradores, 1990).

Os minérios superficiais da jazida de Tapira apresentam diferentes graus de alteração da apatita e minerais de gan- ga, os quais são fatores complicadores na flotação desses fosfatos, a qual é feita utilizando ácidos graxos derivados de óleo de soja e amido de milho, como coletor e depressor, respectivamente. A alteração da apatita pode ocorrer pela substituição do íon cálcio da apatita por cátions bivalentes e/ou formação de minerais fosfáticos secundários. A solubilização da apatita e posterior reprecipitação com outros íons provoca não só a diluição do teor de fósforo em relação ao mineral de origem como também mudanças no sistema cristalográfico e alterações superficiais (Assis e colaboradores, 1990; Salum e colaboradores, 1990).

Lima e Peres (1994) estudaram o comportamento de dois minérios carbonáticos da jazida de Tapira (piroxenito e silexito) na flotação e verificaram que os melhores resultados foram obtidos com uma mistura de ácidos graxos derivados de óleo de arroz com reagentes sintéticos (sulfossuccinato e sulfossuccinamato).

A composição química média dos concentrados de rocha fosfática, produzidos pela Fosfertil, no ano de 2000, foi a seguinte: teores em termos percentuais de $\mathrm{P}_{2} \mathrm{O}_{5}(35,28), \mathrm{Fe}_{2} \mathrm{O}_{3}(1,34), \mathrm{MgO}(0,56)$, $\mathrm{CaO}(50,94)$, relação $\mathrm{CaO} / \mathrm{P}_{2} \mathrm{O}_{5}(1,444)$ (Fosfertil, 2001). Como a mina de TapiraMG possui diversas tipologias de minério, faz-se necessário estudar separadamente o comportamento desses minérios em relação à resposta dos mesmos à flotação, que é o método de concentração empregado industrialmente. Logo, esse trabalho teve por objetivo verificar o comportamento de quatro tipologias de minérios fosfáticos de Tapira-MG, denominados piroxenito silicificado semicompacto, piroxenito silicificado compacto, silexito e piroxenito serpentinizado, utilizando o coletor óleo de soja e o amido de milho como depressor.

\section{Materiais e métodos}

Na Tabela 1, estão apresentados os resultados das análises químicas, que foram realizadas no Laboratório Químico da Fosfertil, pelo método de espectrometria de raios X. Através dessas análises, observa-se que o minério piro- xenito serpentinizado é o de maior teor de $\mathrm{P}_{2} \mathrm{O}_{5}$ e de $\mathrm{CaO}$ e, menor relação $\mathrm{CaO} /$ $\mathrm{P}_{2} \mathrm{O}_{5}(1,31)$. As outras três tipologias de minério possuem baixos teores de $\mathrm{P}_{2} \mathrm{O}_{5} \mathrm{e}$ elevados teores de $\mathrm{CaO}$, ou seja, são minérios de alta relação $\mathrm{CaO} / \mathrm{P}_{2} \mathrm{O}_{5}$.

Os ensaios de flotação realizados consistiram de uma etapa rougher de flotação aniônica direta da apatita. Foi utilizada uma célula Denver do Laboratório de Tratamento de Mineiros do CEFETOP. Os reagentes utilizados foram:

- Coletor: ácido graxo derivado de óleo de soja parcialmente hidrogenado (marca de fantasia Hidrocol e fabricado pela Hidrovex), saponificado com hidróxido de sódio a 5\% p/v.

- Depressor: amido de milho comercial (fabricado pela Cargill), gelatinizado com hidróxido de sódio a $2 \%$ p/v, relação amido/soda $=6 / 1$.

- Soluções de $\mathrm{NaOH}$ ou $\mathrm{HCl}$ a $1 \%$ p/v para ajuste do $\mathrm{pH}$ na faixa de 8 a 11 .

Com base no procedimento-padrão da Fosfertil para a flotação de minérios fosfáticos, foram selecionadas as variáveis consideradas de maior importância, bem como a faixa de trabalho mais indicada. Os fatores foram associados às codificações dos geradores, de forma a evitar se confundirem duas interações prováveis, conforme apresentado na Tabela 2. Para análise dos resultados, foram adotados como variáveis de resposta, o teor $(\mathrm{X})$ e a recuperação de $\mathrm{P}_{2} \mathrm{O}_{5}$ (Y) no concentrado e a relação $\mathrm{CaO} /$ $\mathrm{P}_{2} \mathrm{O}_{5}(\mathrm{Z})$, que representam a qualidade do produto.

Nesse trabalho, o desvio-padrão foi utilizado para quantificar o grau de precisão ou reprodutibilidade das medidas. O erro experimental foi determinado através de quatro experimentos realizados em condições idênticas, utilizando níveis intermediários aos definidos para as variáveis, ver Tabela 2. Os resultados do planejamento fatorial de experimentos foram avaliados, utilizando o algoritmo de Yates, que permite quantificar a significância dos efeitos de cada uma das variáveis estudadas e das interações entre elas. 
Gilberto Caixeta Guimarães et al.

Tabela 1 - Composição química global das tipologias de minério fosfático de Tapira-MG.

\begin{tabular}{l|c|c|c|c|c|c|c|c}
\hline \multirow{2}{*}{ Tipologia } & \multicolumn{7}{c|}{ Teores (\%) } & \multirow{2}{*}{$\begin{array}{c}\text { Relação } \\
\mathbf{C a O}_{\mathbf{\mathbf { P } _ { 2 }}} \mathbf{O}_{\mathbf{5}}\end{array}$} \\
\cline { 2 - 10 } & $\mathbf{P}_{\mathbf{2}} \mathbf{O}_{\mathbf{5}}$ & $\mathbf{F e}_{\mathbf{2}} \mathbf{O}_{\mathbf{3}}$ & $\mathbf{M g O}$ & $\mathbf{C a O}$ & $\mathbf{A l}_{\mathbf{2}} \mathbf{O}_{\mathbf{3}}$ & $\mathbf{S i O}_{\mathbf{2}}$ & $\mathbf{T i O}_{\mathbf{2}}$ & \\
\hline Piroxenito silicificado semi-compacto & 5,29 & 4,32 & 4,20 & 11,03 & 2,31 & 32,09 & 6,21 & 2,10 \\
\hline Piroxenito silicificado sompacto & 7,25 & 4,72 & 3,61 & 22,28 & 1,00 & 22,94 & 4,70 & 3,10 \\
\hline Silexito & 8,43 & 4,03 & 4,57 & 20,87 & 0,42 & 18,81 & 13,55 & 2,50 \\
\hline Piroxenito serpentinizado & 15,90 & 14,39 & 3,20 & 20,86 & 2,75 & 15,67 & 15,63 & 1,31 \\
\hline
\end{tabular}

Tabela 2 - Fatores e níveis selecionados associados à codificação dos geradores.

\begin{tabular}{l|c|c|c|c}
\hline \multirow{2}{*}{ Fator } & \multicolumn{3}{c|}{ Níveis } & \multirow{2}{*}{$\begin{array}{c}\text { Codificação dos } \\
\text { geradores }\end{array}$} \\
\cline { 2 - 5 } & - & + & Intermediário & A \\
\hline Dosagem de coletor (ácido graxo) (g/t) & 300 & 1200 & 750 & $\mathrm{~B}$ \\
\hline Dosagem de depressor (amido de milho) (g/t) & 300 & 700 & 500 & $\mathrm{C}$ \\
\hline $\mathrm{pH}$ na flotação & 8 & 11 & 9,5 & \\
\hline
\end{tabular}

Através dos resultados do planejamento fatorial de experimentos, verificou-se que, para os minérios piroxenito silicificado semicompacto, piroxenito silicificado compacto e silexito, somente a variável dosagem de óleo de soja foi significativa. Desta forma, fixou-se a dosagem de amido em $700 \mathrm{~g} / \mathrm{t}$ e o valor de $\mathrm{pH}$ em 11 para o minério piroxenito silicificado semicompacto, fixou-se a dosagem de amido em $300 \mathrm{~g} / \mathrm{t}$ e o valor de $\mathrm{pH}$ em 8 para o minério piroxenito silicificado compacto e fixou-se a dosagem de amido em $700 \mathrm{~g} / \mathrm{t}$ e o valor de $\mathrm{pH}$ em 11 para o silexito, a fim de otimizar essa variável. Para a outra tipologia de minério (piroxenito serpentinizado), efetuou-se um segundo planejamento de experimentos, onde se fixou o valor de $\mathrm{pH} \mathrm{em} \mathrm{11,} \mathrm{e} \mathrm{se} \mathrm{fixaram}$ dois níveis para o coletor $(200$ e $500 \mathrm{~g} / \mathrm{t}$ ) e fixaram-se dois níveis para o depressor $(800$ e $1000 \mathrm{~g} / \mathrm{t})$.

\section{Resultados e discussão}

Nas Figuras 1, 2, 3, e 4 estão apresentadas a influência da dosagem do óleo de soja sobre o teor, a recuperação de $\mathrm{P}_{2} \mathrm{O}_{5}$ e a relação $\mathrm{CaO} / \mathrm{P}_{2} \mathrm{O}_{5}$ para os minérios piroxenito silicificado semicompacto, piroxenito silicificado compacto e silexito.

Como pode ser observado nas Figuras 1,2 e 3 , o teor de $\mathrm{P}_{2} \mathrm{O}_{5}$ nos concentrados dos minérios piroxenito silicificado semicompacto, piroxenito compacto e silexito diminuiu e a recuperação aumentou com a elevação da dosagem do coletor. Esse comportamento é normal e pode ser atribuído à queda de seletividade na flotação entre a apatita e a ganga a ela associada em função do excesso de coletor presente.

Pela Figura 2, observa-se que a dosagem de $75 \mathrm{~g} / \mathrm{t}$ de óleo de soja foi insuficiente para flotar o minério piroxenito silicificado compacto, pois, tanto o teor, quanto a recuperação, nessa dosagem, foram inferiores aos valores obtidos para as maiores dosagens bem como foi maior a relação $\mathrm{CaO} / \mathrm{P}_{2} \mathrm{O}_{5}$ (Figura 4 - curva PSC).

De uma maneira geral, observa-se que os teores de $\mathrm{P}_{2} \mathrm{O}_{5}$ dos concentrados fori maior para os minérios mais ricos e que a relação $\mathrm{CaO} / \mathrm{P}_{2} \mathrm{O}_{5}$ foram maiores para os minérios com maiores conteúdos de carbonatos, veja as curvas das Figuras 1, 2, 3 e 4, evidenciando, portanto, a baixa seletividade do coletor óleo de soja na flotação da apatita e dos carbonatos presentes nesses minérios. $\mathrm{Ob}-$ serva-se, também, uma elevação da relação $\mathrm{CaO} / \mathrm{P}_{2} \mathrm{O}_{5}$ com o aumento da dosagem do coletor para todas as três tipologias (PSSC- piroxenito silicificado semicompacto, PSC - piroxenito silicificado compacto e, SILE- silexito). Fica clara, pelas curvas apresentadas nas Figuras $1,2,3$ e 4 , a necessidade de etapas de limpeza dos concentrados produzidos para a obtenção de concentrados finais dentro das especificações comerciais.

Para a etapa "rougher", pode-se considerar satisfatória recuperação acima de $90 \%$, pois, nessa etapa, a mesma é priorizada. Dessa forma, pode-se dizer que o ensaio para o minério piroxenito silicificado semicompacto, usando a dosagem de $250 \mathrm{~g} / \mathrm{t}, 700 \mathrm{~g} / \mathrm{t}$ de depressor e $\mathrm{pH}$ igual a 11, foi o que produziu o melhor resultado, pois, mesmo com o au- 


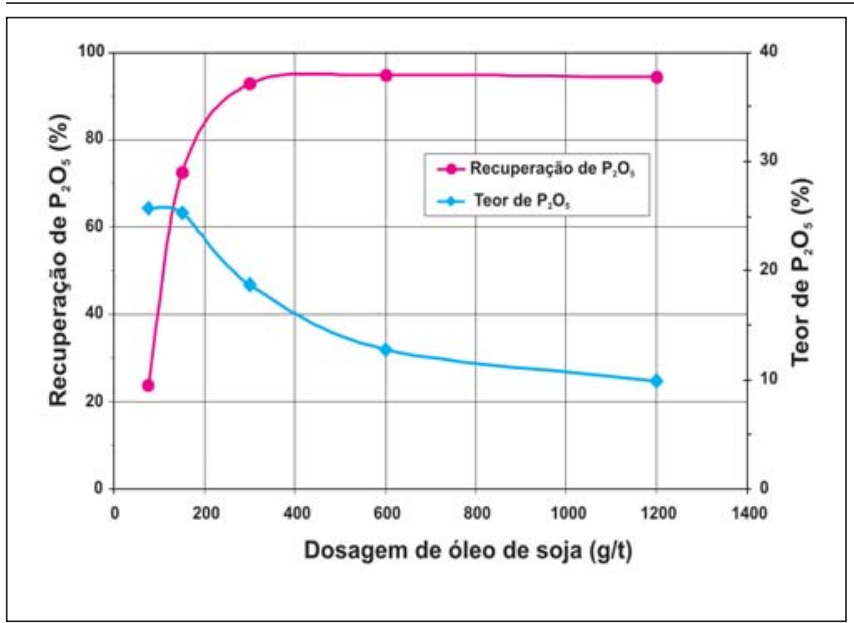

Figura 1 - Influência da dosagem de coletor sobre o teor e a recuperação de $\mathrm{P}_{2} \mathrm{O}_{5}$ do minério piroxenito silicificado semicompacto para a dosagem de amido igual a $700 \mathrm{~g} / \mathrm{t} \mathrm{e} \mathrm{pH} 11$.

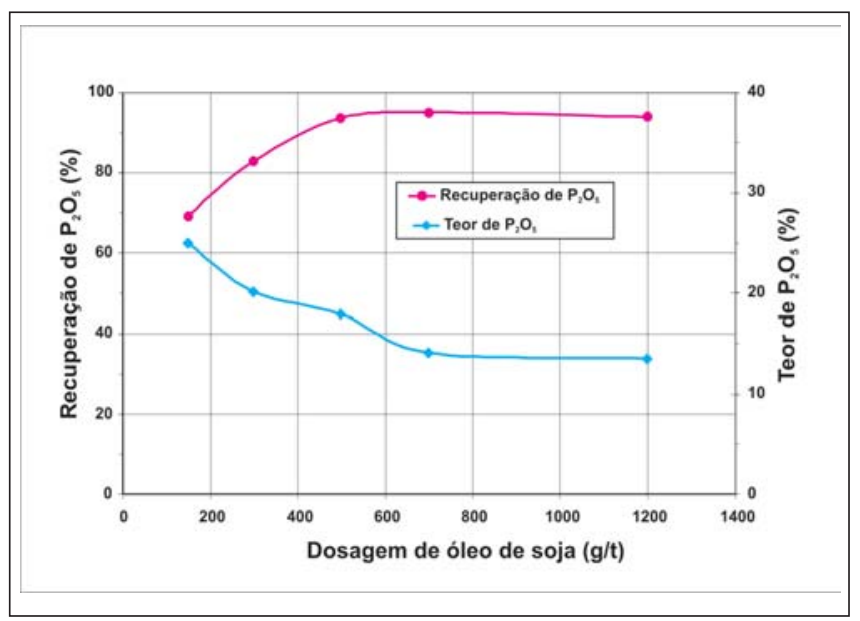

Figura 3 - Influência da dosagem de coletor sobre o teor e a recuperação de $\mathrm{P}_{2} \mathrm{O}_{5}$ do minério silexito para a dosagem de amido igual a $700 \mathrm{~g} / \mathrm{t} \mathrm{e} \mathrm{pH} 11$.

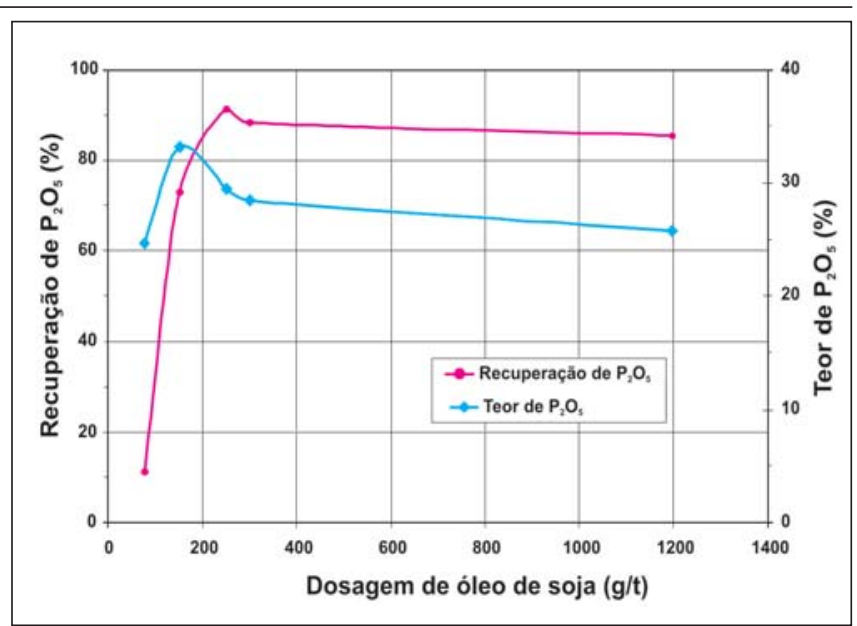

Figura 2 - Influência da dosagem de óleo de soja sobre o teor e a recuperação de $\mathrm{P}_{2} \mathrm{O}_{5}$ do minério piroxenito silicificado compacto para a dosagem de amido igual a $300 \mathrm{~g} / \mathrm{t} \mathrm{e} \mathrm{pH} 8$.

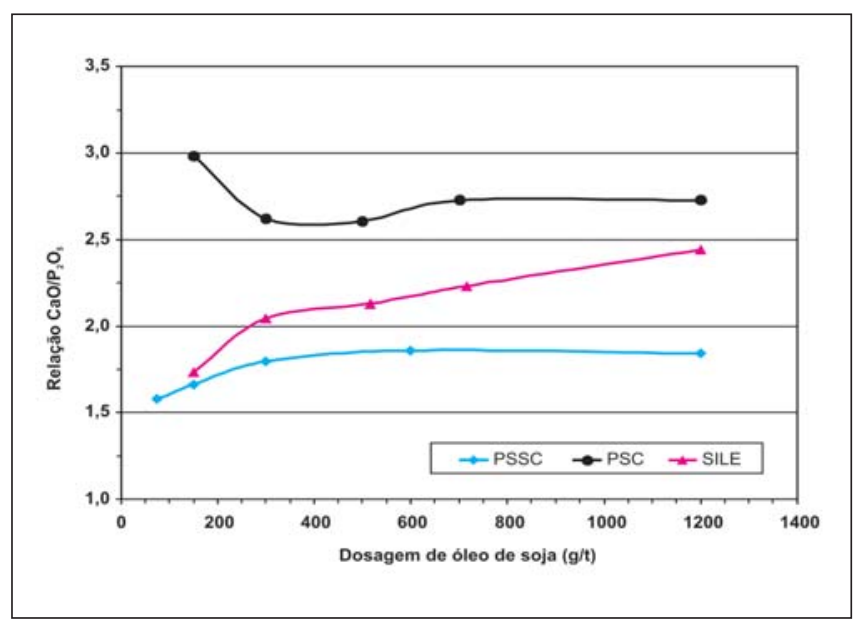

Figura 4 - Influência da dosagem de coletor sobre a relação $\mathrm{CaO} / \mathrm{P}_{2} \mathrm{O}_{5}$ no concentrado do minério piroxenito silicificado semicompacto - PSSC (amido $=700 \mathrm{~g} / \mathrm{t}, \mathrm{pH}=11$ ), piroxenito silicificado compacto $-\mathrm{PSC}$ (amido $=300 \mathrm{~g} / \mathrm{t}, \mathrm{pH}=8)$ e silexito - SILE $($ amido $=700 \mathrm{~g} / \mathrm{t}, \mathrm{pH}=11)$. mento da dosagem de coletor, não se observou um aumento substancial na recuperação de $\mathrm{P}_{2} \mathrm{O}_{5}$ a partir dessa dosagem. Nessas condições, foram obtidos uma recuperação e um teor de $\mathrm{P}_{2} \mathrm{O}_{5}$ em torno de $90 \%$ e $20 \%$, respectivamente. A relação $\mathrm{CaO} / \mathrm{P}_{2} \mathrm{O}_{5}$ foi de 1,7, ver Figuras 1 e 4. Para o minério piroxenito silicificado compacto, a condição otimizada foi obtida para as dosagens de óleo de soja e amido de 250 e $300 \mathrm{~g} / \mathrm{t}$, respectivamente, e valor de $\mathrm{pH}$ igual a 8 , onde foram obtidos recuperação e teor de $\mathrm{P}_{2} \mathrm{O}_{5}$ de cerca $92 \%$ e $14 \%$, respectivamente. A relação $\mathrm{CaO} / \mathrm{P}_{2} \mathrm{O}_{5}$ foi de 2,7 (ver Figura 2 e 4). Para o minério silexito, foi obtida recuperação de $\mathrm{P}_{2} \mathrm{O}_{5}$ igual $90 \%$, teor de $\mathrm{P}_{2} \mathrm{O}_{5}$ igual a $18 \%$ e relação $\mathrm{CaO} / \mathrm{P}_{2} \mathrm{O}_{5}$ de 2,3 para a dosagem de óleo de soja igual a $500 \mathrm{~g} / \mathrm{t}$, amido igual a $700 \mathrm{~g} / \mathrm{te} \mathrm{pH}$ igual a 11 .

$\mathrm{Na}$ Tabela 3, estão apresentados os resultados dos ensaios de flotação para o minério piroxenito serpentinizado. Os melhores resultados alcançados para essa tipologia de minério foram para dosagens de coletor e de depressor de 500 e $800 \mathrm{~g} / \mathrm{t}$, respectivamente, e valor de $\mathrm{pH}$ igual a 11. Nessas condições, foi obtido um concentrado com teor de $\mathrm{P}_{2} \mathrm{O}_{5}$ de $33,0 \%$, recuperação de $\mathrm{P}_{2} \mathrm{O}_{5}$ de $92,69 \%$ e relação $\mathrm{CaO} / \mathrm{P}_{2} \mathrm{O}_{5}$ de 1,4 , que já se encontrava próximo às especifica- ções dos concentrados produzidos pela Fosfertil.

\section{Conclusões}

$\mathrm{O}$ melhor valor de $\mathrm{pH}$ para a flotação dos minérios piroxenito silicificado semicompacto, silexito e serpentinito foi 11 e 8 para o piroxenito silicificado.

Observou-se que os teores de $\mathrm{P}_{2} \mathrm{O}_{5}$ dos minérios mais pobres foram menores e que as relações $\mathrm{CaO} / \mathrm{P}_{2} \mathrm{O}_{5}$ foram maiores para os minérios de maiores conteúdos de carbonatos, evidenciando a baixa seletividade do coletor óleo de soja na flotação desses minérios. 
Gilberto Caixeta Guimarães et al.

Tabela 3 - Piroxenito serpentinizado (serpentinito); coletor: ácido graxo; depressor: amido de milho; $\mathrm{pH}=11$.

\begin{tabular}{c|c|l|c|c}
\hline \multicolumn{2}{c|}{ Reagentes $(\mathbf{g} / \mathbf{t})$} & \multicolumn{2}{c|}{$\mathbf{P}_{\mathbf{2}} \mathbf{O}_{\mathbf{5}}$ no concentrado $(\%)$} & \multirow{2}{*}{ Relação $\mathrm{CaO}_{\mathbf{2}} \mathbf{P}_{\mathbf{2}} \mathbf{O}_{\mathbf{5}}$} \\
\cline { 1 - 3 } Coletor & Depressor & Teor & Recuperação & \\
\hline 200 & 800 & 32,92 & 90,06 & 1,45 \\
\hline 500 & 800 & 33,00 & 92,69 & 1,40 \\
\hline 200 & 1000 & 35,99 & 66,36 & 1,40 \\
\hline 500 & 1000 & 33,53 & 92,57 & 1,43 \\
\hline
\end{tabular}

A melhor dosagem do coletor óleo de soja para a flotação dos minérios piroxenito silicificado semi-compacto e piroxenito silicificado foi $250 \mathrm{~g} / \mathrm{t}$, porém a dosagem de depressor foi de $700 \mathrm{~g} / \mathrm{t}$ para o piroxenito silicificado semicompacto e $300 \mathrm{~g} / \mathrm{t}$ para o piroxenito silicificado compacto.

Ficou evidente a necessidade de etapas de limpeza dos concentrados de todos os minérios estudados. No caso do minério piroxenito serpentinizado, foi obtido, na etapa rougher, um concentrado próximo às especificações de mercado em termos de teor de $\mathrm{P}_{2} \mathrm{O}_{5}(33 \%)$ e relação $\mathrm{CaO} / \mathrm{P}_{2} \mathrm{O}_{5}(1,4)$ com recuperação de $\mathrm{P}_{2} \mathrm{O}_{5}$ superior a $90 \%$.

\section{Referências bibliográficas}

ASSIS, S. M., SILVA, J. M., ARAUJO, A. C. A new approach for the flotation of Brazilian carbonated phosphate ores. In: INTERNATIONAL MINERAL PROCESSING CONGRESS, 16. Amsterdam. Anais..., p. 1561-1572. 1988.

ASSIS, S. M., SALUM. M. J. G., MELLO, M. O., BARROS, L. A. F. Caracterização da performance na flotação de um fosfato alterado de Tapira, MG. In: ENCONTRO NACIONAL DE TRATAMENTO DE MINÉRIOS E HIDROMETALURGIA, 14. Salvador. Anais..., p. 323-336. 1990. BARROS, L. A. F. Flotação da apatita da jazida de Tapira-MG. São Paulo: Escola
Politécnica, Universidade de São Paulo, 1997. 106p. (Dissertação de Mestrado). FOSFERTIL Complexo de Mineração de Tapira (CMT). Tapira: 2001. 17p. (Dados Gerais).

LEAL FILHO. L. S., PERES, A. E. C., OLIVEIRA, R. N., DAMASCENO, A. H. T. Estudos de novos depressores para a flotação direta do fosfato de Jacupiranga. In: ENCONTRO NACIONAL DE MINÉRIOS E HIDROMETALURGIA, 14. Salvador. Anais..., p. 422-450. 1990.

LIMA, R. M. F., PERES, A. E. C. Flotation of refractory phosphate ores. In: Flotation, a volume in memory of Alexander Sotulov, Ed. S. Castro, Concepción. Anais..., p. 207-213. 1994.

Artigo recebido em 11/05/2004 e aprovado em 14/09/2004.

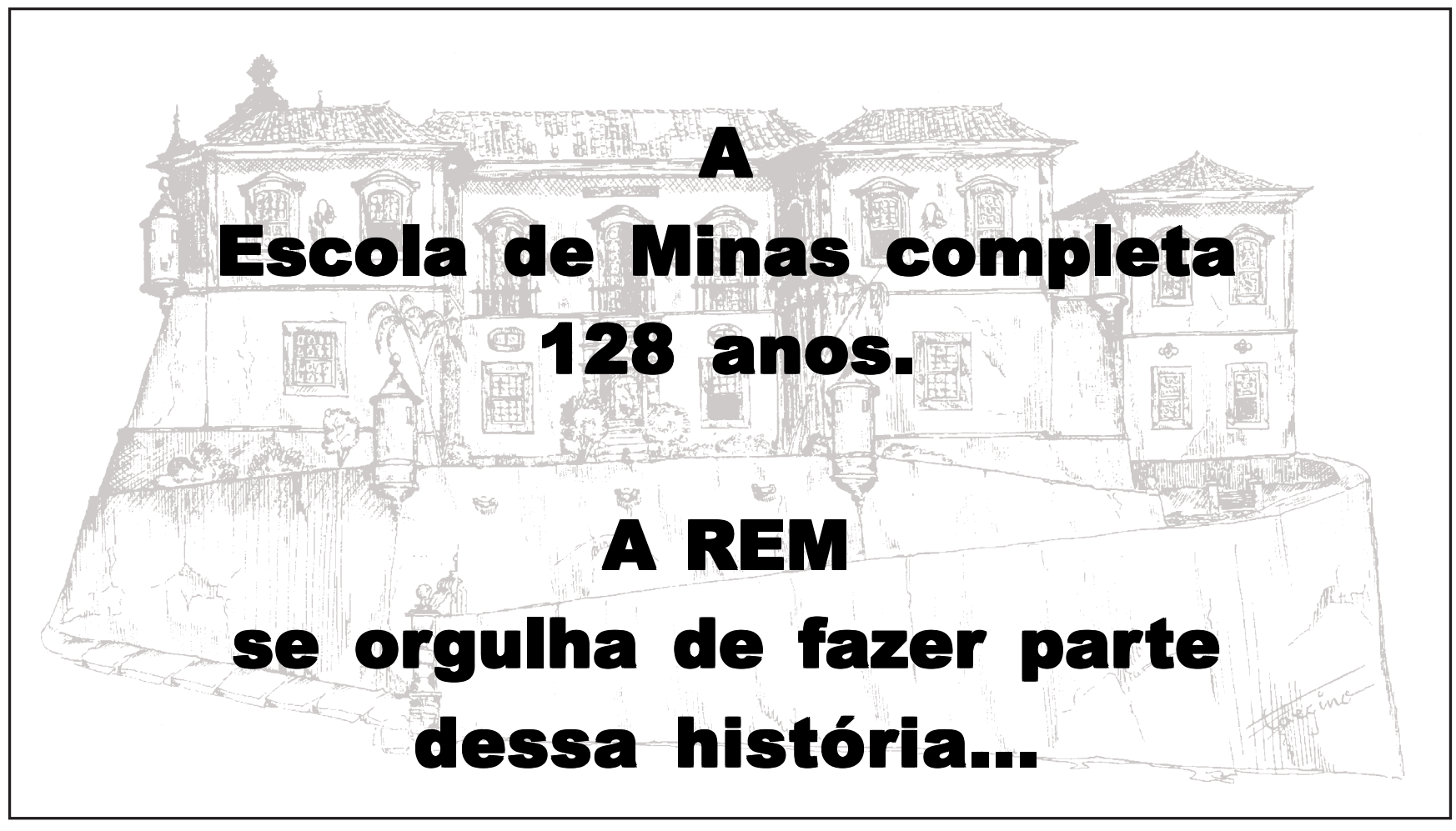

